\title{
Realization of universal quantum cloning with SQUID qubits in a cavity
}

\author{
Jian Yang, Ya-Fei Yu, and Zhi-Ming Zhang* \\ Laboratory of Photonic Information Technology, School of Information and Photonelectronic Science and Engineering, \\ South China Normal University, Guangzhou 510006, China.
}

(Dated: November 25, 2018)

\begin{abstract}
We propose a scheme to realize $1 \rightarrow 2$ universal quantum cloning machine (UQCM) with superconducting quantum interference device (SQUID) qubits, embeded in a high-Q cavity. CNOT operations are derived to present our scheme, and the two-photon Raman resonance processes are used to increase the operation rate. Compared with previous works, our scheme has advantages in the experimental realization and further utilization.
\end{abstract}

PACS numbers: 03.67.Lx, 85.25.Dq, 42.50.Pq, 42.50.Dv

\section{INTRODUCTION}

The field of quantum computation and quantum information has grown so rapidly in the recent years [1]. Quantum mechanics provides us powerful tools to solve specific problems, while it also imposes extra limitations. Duplication is one of the most common operations on the classical computer, however, the so-called quantum no cloning theorem tells us that one can not copy arbitrary unknown quantum states perfectly [2]. However, several schemes have been proposed to achieve realistic quantum cloning machines, which generate copies of arbitrary input qubits approximately, or probabilistically $[3-8]$.

The universal quantum cloning machine (UQCM) is first discussed by Buzĕk and Hillery [4], which provides identical fidelities to arbitrary input qubits. The progress on quantum cloning is reviewed by Scarani et al. [9]. The optimal $1 \rightarrow 2$ UQCM performs the transformations:

$$
\begin{aligned}
& |+\rangle|\Sigma\rangle \rightarrow \sqrt{\frac{2}{3}}|+\rangle|+\rangle\left|A_{\perp}\right\rangle+\sqrt{\frac{1}{3}}|\Phi\rangle|A\rangle, \\
& |-\rangle|\Sigma\rangle \rightarrow \sqrt{\frac{2}{3}}|-\rangle|-\rangle|A\rangle+\sqrt{\frac{1}{3}}|\Phi\rangle\left|A_{\perp}\right\rangle,
\end{aligned}
$$

where qubits are encoded in the basis $\{| \pm\rangle\}$, while $|\Sigma\rangle$ is the initial state of blank copies and ancilla qubits, $\left|A_{\perp}\right\rangle$ and $|A\rangle$ are the final ancilla states, and $|\Phi\rangle=$ $(|+\rangle|-\rangle+|-\rangle|+\rangle) / \sqrt{2}$. After this operation, each copies has a fidelity of $5 / 6$ [4], when compared to the input state. Based on cavity QED, Milman et al. [10] and Zou et al. [11] have proposed different schemes to realize UQCM. However, at least two cavities or two cavity-modes are needed in these works. Inspired by paper [12], we turn to construct UQCM with SQUID embed in a high-Q cavity, based on which, many quantum information processing schemes have been proposed [13-15]. Our motivation is as follows:

\footnotetext{
${ }^{*}$ Corresponding author. Electronic address: zmzhang@scnu.edu.cn
}

(1) Unlike Rydberg atom used in cavity-QED, which is a "flying" qubit, SQUID embed in a high-Q cavity is "static", so it is comparatively easy to adjust its coupling coefficient to the cavity field, or to manipulate it with classical field or quantized field. In our scheme, only one cavity is needed, which accords with the current experimental conditions.

(2) The level spacing of SQUID can be adjusted by changing the external flux or the critical current [12], so we can easily "turn-on" or "turn-off" the interaction between the SQUID and the cavity field. Atomvelocity-selected device and passing qubits detection are not needed in our scheme.

This paper is organized as follows. In Sec. II, we review the SQUID driven by quantized or classical microwave fields. CNOT gate and two specific processes are also constructed. In Sec. III, we discuss the detail of realizing the UQCM. A brief discussion and conclusions are presented in Sec. IV.

\section{MANIPULATION OF SQUID}

We assume that the SQUID, considered throughout this paper, has a $\Lambda$-type three-level structure, as shown in FIG.1. The SQUID qubit is embed in a high-Q cavity and can be manipulated by the cavity field as well as the microwave pulses. In this section, we review the effective Hamiltonian of the SQUID driven by a quantized or classical field. More details of SQUID and Josephsonjunction are discussed by Makhlin et al. [16]. Specific processes as well as CNOT gate are also derived to realize the UQCM.

\section{A. Resonant interaction between SQUID and a cavity field.}

Consider a $\Lambda$-type three-level SQUID embeded in a high-Q cavity. If its $|g\rangle \leftrightarrow|e\rangle$ transition is resonant with the cavity field, the Hamiltonian in the interaction picture under rotating-wave approximation can be written 
as [12]:

$$
H_{I}=\lambda\left[a^{\dagger}|g\rangle\langle e|+a| e\rangle\langle g|\right] .
$$

where $\lambda$ is the effective coupling constant [12], and $a^{\dagger}$ and $a$ are the creation and annihilation operator of the cavity field, respectively. The evolution of this system can be easily derived as:

$$
\begin{aligned}
& |g, 1\rangle \rightarrow \cos (\lambda t)|g, 1\rangle-i \sin (\lambda t)|e, 0\rangle \\
& |e, 0\rangle \rightarrow \cos (\lambda t)|e, 0\rangle-i \sin (\lambda t)|g, 1\rangle
\end{aligned}
$$

\section{B. Resonant interaction between SQUID and a classical microwave pulse}

Consider a $\Lambda$-type three-level SQUID driven by a classical microwave pulse and suppose its $|g\rangle \leftrightarrow|e\rangle$ transition is resonant with the classical field. Then the effective Hamiltonian in the interaction picture under rotatingwave approximation can be written as [12]:

$$
H_{I}=\Omega_{g e}(|g\rangle\langle e|+| e\rangle\langle g|),
$$

where $\Omega_{g e}$ is the effective coupling constant. The evolution of this system can be written as [12]:

$$
\begin{aligned}
& |g\rangle \rightarrow \cos \left(\Omega_{g e} t\right)|g\rangle-i \sin \left(\Omega_{g e} t\right)|e\rangle, \\
& |e\rangle \rightarrow \cos \left(\Omega_{g e} t\right)|e\rangle-i \sin \left(\Omega_{g e} t\right)|g\rangle .
\end{aligned}
$$

Similarly, if the $|i\rangle \leftrightarrow|e\rangle$ transition is resonant with the microwave pulse, and the other transitions are far-off resonant, the evolution of SQUID can be written as [12]:

$$
\begin{aligned}
& |i\rangle \rightarrow \cos \left(\Omega_{i e} t\right)|i\rangle-i \sin \left(\Omega_{i e} t\right)|e\rangle, \\
& |e\rangle \rightarrow \cos \left(\Omega_{i e} t\right)|e\rangle-i \sin \left(\Omega_{i e} t\right)|i\rangle .
\end{aligned}
$$

where $\Omega_{i e}$ is the effective coupling constant.

\section{Interaction between SQUID and two microwave pulses with large detuning}

Consider a $\Lambda$-type three-level SQUID driven by two classical microwave pulses 1 and 2 (depicted in FIG. 1). This system constructs a two-photon Raman resonance, if the $|g\rangle \leftrightarrow|e\rangle$ transition and the $|i\rangle \leftrightarrow|e\rangle$ transition are coupled to the microwave pulses 1 and 2 with identical detunings, i.e. $\Delta=\omega_{g e}-\omega_{1}=\omega_{i e}-\omega_{2}$, where $\omega_{1}$ and $\omega_{2}$ are the frequencies of two microwave pulses. In the case of large detuning, the upper state $|e\rangle$ can be eliminated adiabatically, and the evolution of this system follows [17]:

$$
\begin{aligned}
|g\rangle \rightarrow & \cos \left(\lambda^{\prime} t\right)|g\rangle \\
& +e^{-i \omega_{g i} t} e^{-i\left(\phi_{1}-\phi_{2}-\pi / 2\right)} \sin \left(\lambda^{\prime} t\right)|i\rangle, \\
|i\rangle \rightarrow & e^{i\left(\phi_{1}-\phi_{2}+\pi / 2\right)} \sin \left(\lambda^{\prime} t\right)|g\rangle \\
& +e^{-i \omega_{g i} t} \cos \left(\lambda^{\prime} t\right)|i\rangle,
\end{aligned}
$$

where $\phi_{1}$ and $\phi_{2}$ are the initial phases of two classical microwave pluses, respectively, and $\lambda^{\prime}$ is the effective coupling constant.

Now we turn to construct two processes based on Eq. (7), which are further needed in our universal cloning scheme.

Process 1: In this process, we want to realize the transformation:

$$
\begin{aligned}
& |+\rangle \rightarrow-|i\rangle, \\
& |-\rangle \rightarrow|g\rangle,
\end{aligned}
$$

where $| \pm\rangle=(|i\rangle \pm|g\rangle) / \sqrt{2}$, and this can be realized with two steps:

Step (1): Apply two microwave pulses to SQUID with $\phi_{1}-\phi_{2}=3 \pi / 2$, and adjust the interaction time to be $t_{1}=3 \pi / 4 \lambda^{\prime}$.

Step (2): Turn off the classical pulse, and let the SQUID undergo a free evolution for a time $t_{2}$, such that $\omega_{g i}\left(t_{1}+t_{2}\right)=2 m \pi$ ( $m$ is an integer $)$.

This process can be detailed as:

$$
\begin{aligned}
|g\rangle & \rightarrow \frac{-1}{\sqrt{2}}|g\rangle-\frac{e^{-i \omega_{g i} t_{1}}}{\sqrt{2}}|i\rangle \\
& \rightarrow \frac{-1}{\sqrt{2}}(|i\rangle+|g\rangle), \\
|i\rangle & \rightarrow \frac{1}{\sqrt{2}}|g\rangle-\frac{e^{-i \omega_{g i} t_{1}}}{\sqrt{2}}|i\rangle \\
& \rightarrow \frac{-1}{\sqrt{2}}(|i\rangle-|g\rangle) .
\end{aligned}
$$

Process 2: In this process, we want to construct a transformation, which has the form:

$$
\begin{aligned}
& |g\rangle \rightarrow|-\rangle=\frac{1}{\sqrt{2}}(|i\rangle-|g\rangle), \\
& |i\rangle \rightarrow-|+\rangle=\frac{-1}{\sqrt{2}}(|i\rangle+|g\rangle) .
\end{aligned}
$$

and this can be achieved by two steps:

Step (1): Apply two microwave pulses to SQUID with $\phi_{1}-\phi_{2}=\pi / 2$, and adjust the interaction time to be $t_{1}^{\prime}=3 \pi / 4 \lambda^{\prime}$;

Step (2): Turn off the classical pulse, and let the SQUID undergo a free evolution for a time $t_{2}^{\prime}$, such that $\omega_{g i}\left(t_{1}^{\prime}+t_{2}^{\prime}\right)=2 m \pi$ ( $m$ is an integer $)$.

This process can be summarized as:

$$
\begin{aligned}
& |g\rangle \rightarrow \frac{-1}{\sqrt{2}}|g\rangle+\frac{e^{-i \omega_{g i} t_{1}^{\prime}}}{\sqrt{2}}|i\rangle \rightarrow|-\rangle, \\
& |i\rangle \rightarrow \frac{-1}{\sqrt{2}}|g\rangle-\frac{e^{-i \omega_{g i} t_{1}^{\prime}}}{\sqrt{2}}|i\rangle \rightarrow-|+\rangle,
\end{aligned}
$$

We can adjust the evolution time $t_{2}$ and $t_{2}^{\prime}$ to satisfy $t_{1}+t_{2}=t_{1}^{\prime}+t_{2}^{\prime}=2 m \pi$. In this case, the two processes cost the same time. 


\section{CNOT Gate}

CNOT is one of the most essential operations in quantum information, and it also plays important role in our scheme. Inspired by previous work [18], which presents CNOT gate in the cavity-QED with Rydberg atom, here we propose a scheme to achieve CNOT operation based on the interaction between SQUID and the cavity field, as discussed in Section II A. Suppose that the information is coded on the $|g\rangle$ and $|i\rangle$ levels of the SQUID, which is embed in a high-Q cavity, and its $|g\rangle \leftrightarrow|e\rangle$ transition is set to be resonant with the cavity field. The SQUID is initially in an arbitrary state $|\psi\rangle=\alpha_{s}|+\rangle+\beta_{s}|-\rangle$, and the cavity is prepared in a superposition $|\varphi\rangle=\alpha_{f}|0\rangle+\beta_{f}|1\rangle$, where $| \pm\rangle_{s}=\left(|i\rangle_{s} \pm|g\rangle_{s}\right) / \sqrt{2}$, and $\left|\alpha_{s}\right|^{2}+\left|\beta_{s}\right|^{2}=$ $\left|\alpha_{f}\right|^{2}+\left|\beta_{f}\right|^{2}=1$. The evolution of this system follows Eq. (3), and after a time $t=\pi / \lambda$, we have:

$$
\begin{aligned}
| \pm\rangle_{s}|0\rangle_{f} & \rightarrow| \pm\rangle_{s}|0\rangle_{f}, \\
| \pm\rangle_{s}|1\rangle_{f} & \rightarrow|\mp\rangle_{s}|1\rangle_{f},
\end{aligned}
$$

which corresponds to a CNOT operation. In this scheme, the cavity field is the control qubit, and the SQUID is the target qubit.

\section{UNIVERSAL QUANTUM CLONING MACHINE}

In this section, we turn to describe the detail of the UQCM. Suppose that three identical SQUIDs are embeded in a high-Q cavity (depicted in FIG. 2). To make the couplings between the SQUIDs and the cavity field are the same, we need to adjust the positions of every SQUID such that the magnetic components $\mathbf{B}\left(\mathbf{r}_{1}, t\right), \mathbf{B}\left(\mathbf{r}_{2}, t\right)$, and $\mathbf{B}\left(\mathbf{r}_{3}, t\right)$ of the cavity field, imposed on SQUIDs, are the same. More technical considerations have been discussed in the previous papers [12] 14]. The cavity is initially prepared in the vacuum state $|0\rangle_{f}$, and SQUIDs are in the state $|g\rangle_{1}|g\rangle_{2}|g\rangle_{3}$.

Let SQUID1 be prepared in an arbitrary state $|\psi\rangle_{1}=$ $\alpha|+\rangle_{1}+\beta|-\rangle$ with two classical microwave pulses, where $|\alpha|^{2}+|\beta|^{2}=1$. The UQCM is to achieve the transformation described by Eq. (1).

Step (1): Let SQUID2 be driven by a classical microwave pulse. Following Eq. (5), we have:

$$
|\varphi\rangle_{2}=\sqrt{\frac{2}{3}}|g\rangle_{2}+i \sqrt{\frac{1}{3}}|e\rangle_{2} .
$$

Step (2): Adjust the level spacing of SQUID2 to make its $|g\rangle \leftrightarrow|e\rangle$ transition resonant with the cavity field. Undergoing a $t=\pi / 2 \lambda$ evolution, the state of SQUID2 and the cavity field is changed to:

$$
|\varphi\rangle_{2}|0\rangle_{f} \rightarrow|g\rangle_{2}\left(\sqrt{\frac{2}{3}}|0\rangle_{f}+\sqrt{\frac{1}{3}}|1\rangle_{f}\right) .
$$

Step (3): Turn-off SQUID2 (i.e. making it does not interact with the field), and let the $|g\rangle \leftrightarrow|e\rangle$ transition of SQUID1 be coupled to the cavity field. With a CNOT operation detailed in Eq. (12), the system is changed to:

$$
\begin{aligned}
& \sqrt{\frac{2}{3}}\left(\alpha|+\rangle_{1}+\beta|-\rangle_{1}\right)|g\rangle_{2}|0\rangle_{f}+ \\
& \sqrt{\frac{1}{3}}\left(\alpha|-\rangle_{1}+\beta|+\rangle_{1}\right)|g\rangle_{2}|1\rangle_{f} .
\end{aligned}
$$

Step (4): Turn-off SQUID1, and let the SQUID2 interact with the cavity field again. After a time $t=\pi / 4 \lambda$, the system is changed to

$$
\begin{aligned}
& \sqrt{\frac{2}{3}}\left(\alpha|+\rangle_{1}+\beta|-\rangle_{1}\right)|g\rangle_{2}|0\rangle_{f}+ \\
& \sqrt{\frac{1}{6}}\left(\alpha|-\rangle_{1}+\beta|+\rangle_{1}\right) \cdot\left(|g\rangle_{2}|1\rangle_{f}-i|e\rangle_{2}|0\rangle_{f}\right) .
\end{aligned}
$$

Step (5): Turn-off SQUID2, and let SQUID3 interact with the cavity field for a time $t=\pi / 2 \lambda$. We get:

$$
\begin{aligned}
& {\left[\sqrt{\frac{2}{3}}\left(\alpha|+\rangle_{1}+\beta|-\rangle_{1}\right)|g\rangle_{2}|g\rangle_{3}-\right.} \\
& \left.i \sqrt{\frac{1}{6}}\left(\alpha|-\rangle_{1}+\beta|+\rangle_{1}\right) \cdot\left(|g\rangle_{2}|e\rangle_{3}+|e\rangle_{2}|g\rangle_{3}\right)\right]|0\rangle_{f} .
\end{aligned}
$$

Step (6): Let SQUID2 and SQUID3 be driven with identical classical microwave pulses on its $|g\rangle \leftrightarrow|i\rangle$ transition, respectively. After a time $t=\pi / 2 \Omega_{i e}$, we get:

$$
\begin{aligned}
& \sqrt{\frac{2}{3}}\left(\alpha|+\rangle_{1}+\beta|-\rangle_{1}\right)|g\rangle_{2}|g\rangle_{3}- \\
& \sqrt{\frac{1}{6}}\left(\alpha|-\rangle_{1}+\beta|+\rangle_{1}\right)\left(|g\rangle_{2}|i\rangle_{3}+|i\rangle_{2}|g\rangle_{3}\right) .
\end{aligned}
$$

Step (7): Then we impose Process 1 (described in section II C) on SQUID1, and Process 2 on SQUID2 and SQUID3, simultaneously. The system will be transformed to:

$$
\begin{aligned}
& \sqrt{\frac{2}{3}}\left(-\alpha|i\rangle_{1}+\beta|g\rangle_{1}\right)|-\rangle_{2}|-\rangle_{3}+ \\
& \sqrt{\frac{1}{3}}\left(\alpha|g\rangle_{1}-\beta|i\rangle_{1}\right)|\Phi\rangle_{23}
\end{aligned}
$$

where $|\Phi\rangle_{23}=\left(|+\rangle_{2}|-\rangle_{3}+|-\rangle_{2}|+\rangle_{3}\right) / \sqrt{2}$.

Step (8): Let SQUID1 undergo a $|i\rangle_{1} \rightarrow-i|e\rangle_{1}$ transformation with a classical microwave pulse. Following Eq. (13), we have:

$$
\begin{aligned}
& \sqrt{\frac{2}{3}}\left(\alpha i|e\rangle_{1}+\beta|g\rangle_{1}\right)|-\rangle_{2}|-\rangle_{3}+ \\
& \sqrt{\frac{1}{3}}\left(\alpha|g\rangle_{1}+\beta i|e\rangle_{1}\right)|\Phi\rangle_{23} .
\end{aligned}
$$


Step (9): Let SQUID1 interact with cavity for a time $t=\pi / 2 \lambda$. We have:

$$
\begin{aligned}
& \sqrt{\frac{2}{3}}\left(\alpha|1\rangle_{f}+\beta|0\rangle_{f}\right)|-\rangle_{2}|-\rangle_{3}+ \\
& \sqrt{\frac{1}{3}}\left(\alpha|0\rangle_{f}+\beta|1\rangle_{f}\right)|\Phi\rangle_{23} .
\end{aligned}
$$

Step (10): Make CNOT operations on SQUID2 and SQUID3 respectively, then we have:

$$
\begin{aligned}
& \alpha\left(\sqrt{\frac{2}{3}}|+\rangle_{2}|+\rangle_{3}\left|A_{\perp}\right\rangle+\sqrt{\frac{1}{3}}|\Phi\rangle_{23}|A\rangle\right)+ \\
& \beta\left(\sqrt{\frac{2}{3}}|-\rangle_{2}|-\rangle_{3}|A\rangle+\sqrt{\frac{1}{3}}|\Phi\rangle_{23}\left|A_{\perp}\right\rangle\right),
\end{aligned}
$$

where $\left|A_{\perp}\right\rangle=|g\rangle_{1}|1\rangle_{f}$ and $|A\rangle=|g\rangle_{1}|0\rangle_{f}$. We notice that Eq. (22) accords with the optimal $1 \rightarrow 2$ UQCM.

\section{DISCUSSION AND CONCLUSIONS}

In conclusion, we have proposed a scheme for realizing a $1 \rightarrow 2$ universal quantum cloning machine (UQCM) with superconducting quantum interference device (SQUID) qubits, embeded in a high-Q cavity. The SQUID qubits can be manipulated either by the cavity field, or by classical microwave pulses. The interaction between the $|g\rangle \leftrightarrow|e\rangle$ transition and the cavity field can be easily "turned-on" or "turned-off" by adjusting the level spacings of the SQUID, which can be achieved by changing the external flux $\Phi_{x}$ and the critical current $I_{c}$ [12]. The microwave pulses are controlled by external equipments, and can manipulate SQUID qubits resonantly or with large detuning. In our scheme, the information is encoded on the $|g\rangle$ and $|i\rangle$ levels, and the decay from the level $|i\rangle$ should be very small, since no direct tunneling between the two levels are required 17]. Twophoton Raman resonance processes are used to achieve the $|g\rangle \leftrightarrow|i\rangle$ transition, which has been shown faster than the conventional gate operations [17].

Different from Rydberg atom, the SQUID used in our scheme is a kind of "static qubit", and we have no need to trap the qubits with complex technology. In previous proposals with Rydberg atoms, the atoms flying with a velocity of $v=500 \mathrm{~m} / \mathrm{s}$ [10] are hard to be trapped, so at least two cavities or two field-modes are needed in the those schemes. However, the embed SQUID can be manipulated with the cavity field for several times, as a result, only one cavity is needed in our UQCM scheme. Neither atom-velocity-selected device nor passing qubits detection is needed in our scheme, and the "static" copies can easily be utilized after UQCM, so it has advantages in experimental realization and further utilization.

\section{Acknowledgement}

This work was supported by the National Natural Science Foundation of China under grant numbers 60578055 and 10404007.
[1] M. A. Nielsen and I. L. Chuang, Quantum Computation and Quantum Information (Cambridge University Press, Cambridge, England, 2000).

[2] W. K. Wootters and W. H. Zurek, Nature (London) 299, 802 (1982).

[3] H. Barnum et al., Phys. Rev. Lett. 76, 2818 (1996).

[4] V. Buzěk and M. Hillery, Phys. Rev. A 54, 1844 (1996).

[5] N. Gisin and S. Massar, Phys. Rev. Lett. 79, 2153 (1997).

[6] L. M. Duan and G. C. Guo, Phys. Rev. Lett. 80, 4999 (1998).

[7] D. Bruß et al., Phys. Rev. A 57, 2368 (1998).

[8] A. K. Pati, Phys. Rev. Lett. 83, 2849 (1999).

[9] V. Scarani, S. Iblisdir, and N. Gisin, Rev. Mod. Phys. 77, 1225 (2005).

[10] P. Milman, H. Ollivier, and J. M. Raimond, Phys. Rev. A 67, 012314 (2003).

[11] X. B. Zou, K. Pahlke, and W. Mathis, Phys. Rev. A 67, 024304 (2003).

[12] C. P. Yang and S. I. Chu, Phys. Rev. A 67, 042311 (2003).

[13] C. P. Yang, S. I. Chu, and S. Y. Han, Phys. Rev. Lett 92, 117902 (2004).

[14] X. L. Zhang, K. L. Gao, and M. Feng, Phys. Rev. A 74 024303 (2006).
[15] M. H. S. Amin, A. Y. Smirnov, and A. Maassen van den Brink, Phys. Rev. B 67 100508(R) (2003).

[16] Y. Makhlin, G. Schön, and A. Shnirman, Rev. Mod. Phys. 73, 357 (2001).

[17] C. P. Yang and S. Y. Han, Phys. Lett. A 321, 273 (2004).

[18] J. M. Raimond, M. Brune, and S. Haroche, Rev. Mod. Phys. b73 565 (2001).

Figure captions

FIG.1: The SQUID qubit driven by two classical microwave pulses. The $\Lambda$-type three levels are denoted as $|g\rangle,|i\rangle$ and $|e\rangle$, respectively. The $|g\rangle \leftrightarrow|e\rangle$ and $|i\rangle \leftrightarrow$ $|e\rangle$ transition are coupled to classical microwave pulses 1 and 2 with identical detuning $\Delta$, where $\omega_{1}$ and $\omega_{2}$ are the frequencies of the pulses.

FIG.2: Three identical SQUID qubits are embed in a high-Q cavity. The coupling constants of SQUIDs and the cavity field are adusted to be the same. Every SQUID can be manipulated by the cavity field or by classical microwave pulses independently. 


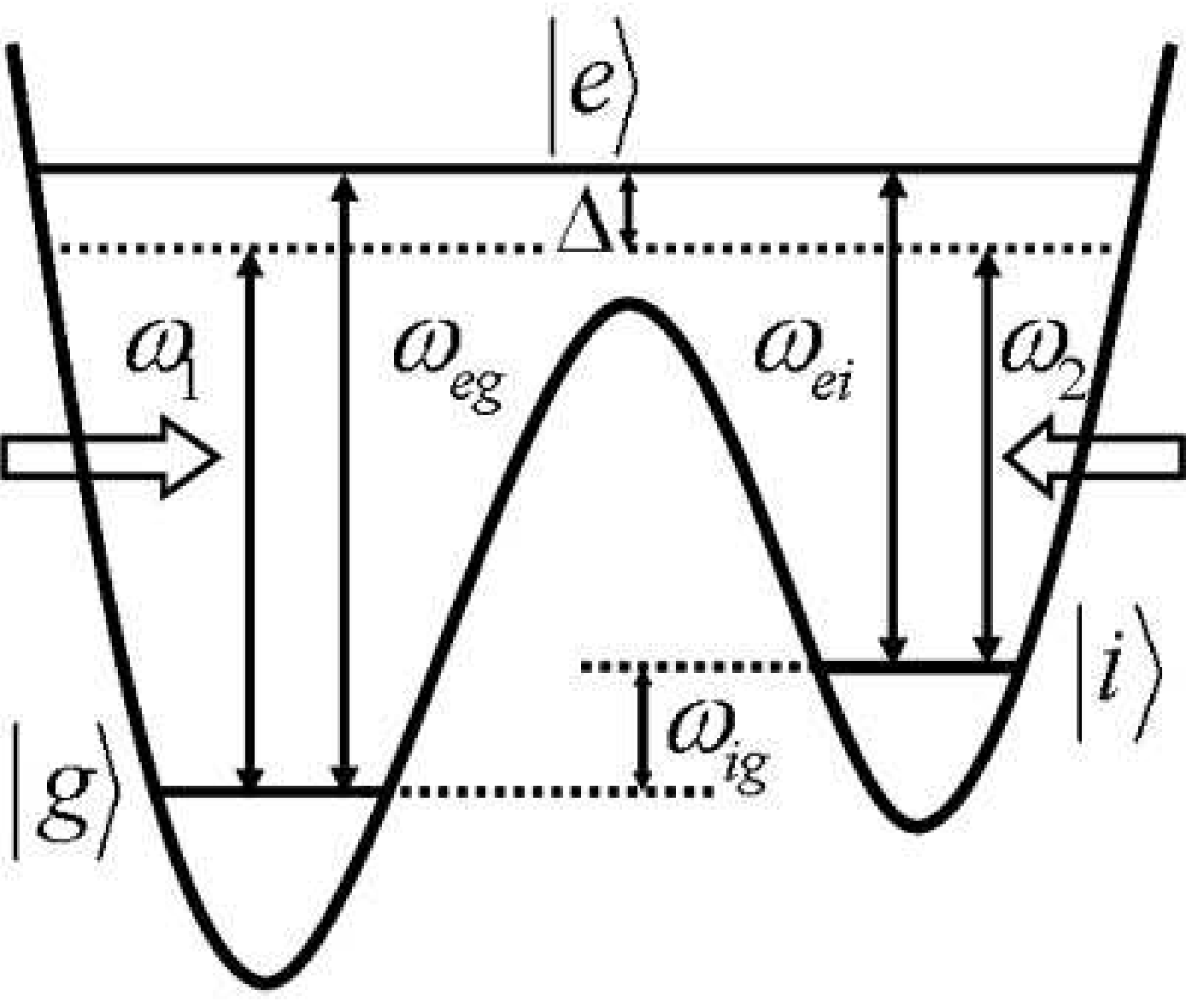




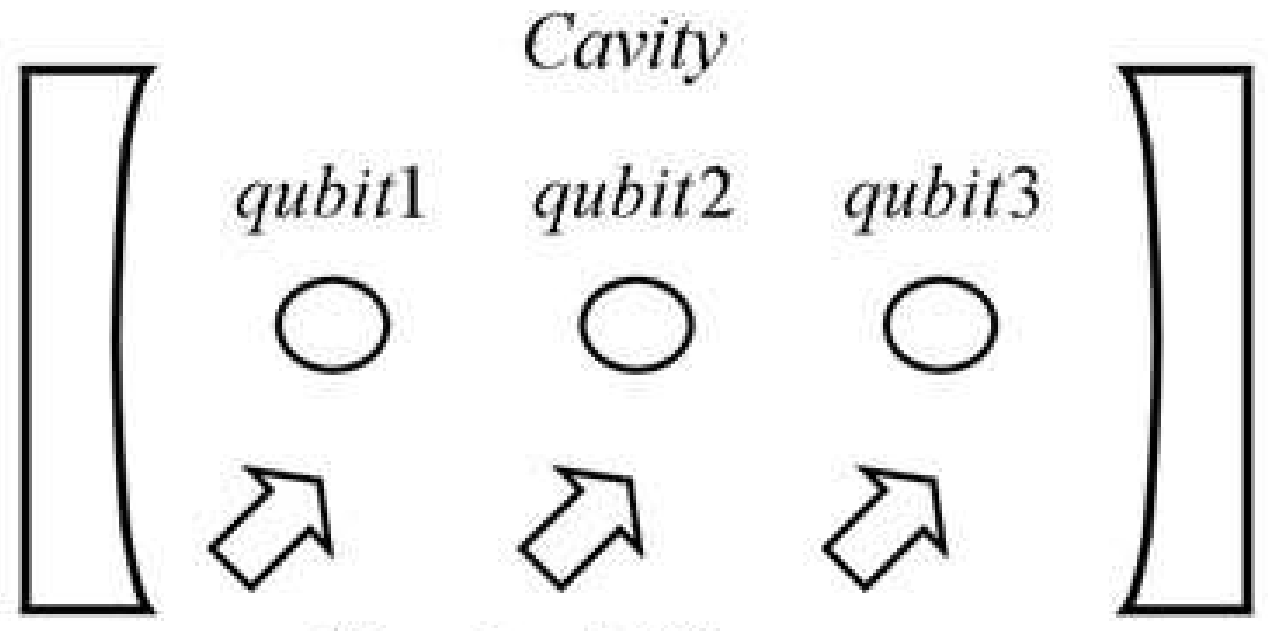

Classical Microwave 